\title{
Clinicopathological predictors of recurrence in nodular and superficial spreading cutaneous melanoma: a multivariate analysis of 214 cases
}

Maria A. Pizzichetta ${ }^{*}$, Daniela Massi ${ }^{2}$, Mario Mandalà ${ }^{3}$, Paola Queirolo ${ }^{4}$, Ignazio Stanganelli ${ }^{5,18}$, Vincenzo De Giorgi ${ }^{6}$, Giovanni Ghigliotti ${ }^{7}$, Stefano Cavicchini ${ }^{8}$, Pietro Quaglino ${ }^{9}$, Maria T. Corradin ${ }^{10}$, Pietro Rubegni ${ }^{11}$, Mauro Alaibac ${ }^{12}$, Stefano Astorino ${ }^{13}$, Fabrizio Ayala ${ }^{14}$, Serena Magi ${ }^{5,18}$, Laura Mazzoni ${ }^{5,18}$, Maria Ausilia Manganoni ${ }^{15}$, Renato Talamini ${ }^{16}$, Diego Serraino ${ }^{16}$, Giuseppe Palmieri ${ }^{17}$ and on behalf of the Italian Melanoma Intergroup (IMI)

\begin{abstract}
Background: Nodular melanoma (NM) accounts for most thick melanomas and because of their frequent association with ulceration, fast growth rate and high mitotic rate, contribute substantially to melanoma-related mortality. In a multicentric series of 214 primary melanomas including 96 NM and 118 superficial spreading melanoma (SSM), histopathological features were examined with the aim to identify clinicopathological predictors of recurrence.

Methods: All consecutive cases of histopathologically diagnosed primary invasive SSM and NM during the period 2005-2010, were retrieved from the 12 participating Italian Melanoma Intergroup (IMI) centers. Each center provided clinico-pathological data such as gender, age at diagnosis, anatomical site, histopathological conventional parameters, date of excision and first melanoma recurrence.

Results: Results showed that NM subtype was significantly associated with Breslow thickness (BT) at multivariate analysis: [BT 1.01-2 mm (OR 7.22; 95\% Cl 2.73-19.05), BT 2.01-4 mm (OR 7.04; 95\% Cl 2.54-19.56), and BT > $4 \mathrm{~mm}(\mathrm{OR}$ $51.78 ; 95 \% \mathrm{Cl} 5.65-474.86)(\mathrm{p}<0.0001)]$. Furthermore, mitotic rate (MR) was significantly correlated with NM histotype: [(MR 3-5 mitoses $/ \mathrm{mm}^{2}$ (OR 2.62; 95\% Cl 1.01-6.83) and MR $>5$ mitoses $/ \mathrm{mm}^{2}$ (OR 4.87; 95\% Cl 1.77-13.40) $(\mathrm{p}=0.002)$ ]. The risk of recurrence was not significantly associated with NM histotype while BT [BT 1.01-2.00 mm (HR 1.55; 95\% Cl 0.51-4.71), BT 2.01-4.00 mm (HR 2.42; 95\% Cl 0.89-6.54), BT > 4.00 mm. (HR 3.13; 95\% Cl 0.95-10.28) ( $p=0.05)]$, mitotic rate $\left[M R>2\right.$ mitoses $\left./ \mathrm{mm}^{2}(\mathrm{HR} 2.34 ; 95 \% \mathrm{Cl}, 1.11-4.97)(\mathrm{p}=0.03)\right]$ and the positivity of lymph node sentinel biopsy (SNLB) (HR 2.60; 95\% Cl 1.19-5.68) ( $p=0.007)$ were significantly associated with an increased risk of recurrence at multivariate analysis.

Conclusions: We found that NM subtype was significantly associated with higher BT and MR but it was not a prognostic factor since it did not significantly correlate with melanoma recurrence rate. Conversely, increased BT and MR as well as SNLB positivity were significantly associated with a higher risk of melanoma recurrence.
\end{abstract}

Keywords: Nodular melanoma, Superficial spreading melanoma, Prognostic indicators, Recurrence

\footnotetext{
*Correspondence: pizzichetta@cro.it

${ }^{1}$ Division of Oncology B, CRO Aviano National Cancer Institute, Via Franco

Gallini 2, 33081 Aviano, Italy

Full list of author information is available at the end of the article
} 


\section{Background}

The incidence of melanoma is increasing worldwide with highest rates in northern Europe, United States and Australia [1]. The most commonly prognostic parameters in the recent AJCC/UICC staging model are breslow thickness (BT), ulceration and microscopic satellite while mitotic rate (MR) is an additional attribute that may be used in decision-making [2]. Nodular melanoma (NM) presenting with vertical growth phase without evidence of an initial radial growth phase, grows faster than melanomas associated with a radial growth phase such as superficial spreading melanoma (SSM) and lentigo malignant melanoma (LMM) [3].

According to the new taxonomy framework, NM may occur in sun-exposed skin without or with low cumulative sun-induced damage (low-CSD) or develop on skin with cumulative sun-induced damage (high-CSD) [4]. NM account for most thick melanomas and because of their frequent association with ulceration, fast growth and high mitotic rate, substantially contribute to melanoma-related mortality [3, 5-7].

LMM and SSM have been found to have a better prognosis than NM, however, when comparing for melanoma thickness, a significant difference between the subtypes was not found [8]. SSM and NM are believed to represent sequential phases of linear progression from radial to vertical growth. Clinical, pathological and epidemiologic evidences suggest, however, that SSM and NM might be the result of independent pathways of tumor development and underlying molecular differences between the two subtypes that may also contribute to the disparate outcomes.

The aims of the current study were to evaluate clinical and histopathological features associated with NM and SSM and to identify independent clinicopathological prognostic factors in a multicentre series of 214 primary melanomas including $96 \mathrm{NM}$ and 118 SSM.

\section{Methods}

The study series included only a limited fraction of the consecutive cases of histopathologically diagnosed primary invasive SSM and NM, presented with the combination of clinical and dermoscopic images and complete clinical history, observed during the period 2005-2010, in 12 Italian Melanoma Intergroup (IMI) centers. In order to guarantee sample homogeneity, only SSM and NM cases in patients with at least 5 years of follow-up, by the end of 2015 were included into the study. Each center provided clinical and pathological data such as gender, age at diagnosis, melanoma site, date of excision, pathological conventional parameters and date of first melanoma recurrence. By the beginning of January 2016, all information from the 12 centers was merged into a database at the Cancer Epidemiology Unit (R.T.) of the Centro di Riferimento Oncologico, Aviano (Italy), with a new identification link to the patient information on clinical features and histopathological diagnosis.

\section{Statistical analyses}

Statistical analysis was performed by means of SAS statistical software 9.1 (SAS Institute Inc., Cary, NC, USA). Odds ratios (ORs) and corresponding 95\% confidence intervals (CIs) were computed by unconditioned logistic regression to evaluate differences in the distribution of histopathological features of NM vs SSM. The significance of OR ( $\beta$ parameter) was tested through Wald Chi square. Statistically significant variables in the univariate analysis were included in the multivariate model. Tests for trend were based on the likelihood-ratio test between the models with and without a linear term for each variable of interest. The probability of recurrence was computed from the date of melanoma diagnosis to the date of first relapse or last follow-up. The curves of probability of recurrence were conducted by the Kaplan-Meier method and the differences were assessed with the logrank test [9]. In addition, the differences were also tested in univariate and multivariate analyses using the Cox proportional hazards model to compute the hazard ratio (HR) and corresponding 95\% confidence interval (CI) [10]. The significance of HR ( $\beta$ parameter) was tested through Wald Chi square. All results were considered statistically significant for values of $\mathrm{p} \leq 0.05$ (two-tailed test).

\section{Results}

Of 214 primary cutaneous melanoma, 96 were NM and 118 were SSM. The study included 214 patients (118 men, 96 women) with a median age of 61 years (range: 21-96 years) for patients with NM and 57 years (range: 16-92) for patients with SSM. The sites of primary melanoma included head and neck $(\mathrm{n}=17)$; limb $(\mathrm{n}=84)$; and trunk $(\mathrm{n}=113)$. We observed no differences between NM and SSM with regard to the distribution by sex, age, sites of melanomas, previous personal or family history of melanoma (data not shown).

Table 1 shows the univariate and multivariate analysis (OR) of main histopathological features of NM vs SSM. Multivariate analysis showed that BT was significantly associated with NM subtype: [BT 1.01-2 mm (OR 7.22; 95\% CI 2.73-19.05), BT 2.01-4 mm (OR 7.04; 95\% CI 2.54-19.56), and BT > $4 \mathrm{~mm}$ (OR 51.78; 95\% CI 5.65474.86) ( $<$ 0.0001)] (Table 1). Furthermore mitotic rate (MR) was significantly correlated with NM histotype: [(MR 3-5 mitoses $/ \mathrm{mm}^{2}$ (OR 2.62; 95\% CI 1.01-6.83) and $\mathrm{MR}>5$ mitoses $/ \mathrm{mm}^{2}$ (OR 4.87; 95\% CI 1.77-13.40) $(\mathrm{p}=0.002)]($ Table 1$)$. 
Table 1 Univariate and multivariate analysis (OR) of some histopathological features of nodular melanoma (NM) versus superficial spreading melanomas (SSMs)

\begin{tabular}{|c|c|c|c|c|}
\hline & $\begin{array}{l}\text { NM } \\
\text { (N. 96) }\end{array}$ & $\begin{array}{l}\text { SSMs } \\
\text { (N. 118) }\end{array}$ & $\begin{array}{l}\text { Univariate } \\
\text { OR }(95 \% \mathrm{Cl})^{\mathrm{a}}\end{array}$ & $\begin{array}{l}\text { Multivariate } \\
\text { OR }(95 \% \mathrm{Cl})^{\mathrm{a}}\end{array}$ \\
\hline \multicolumn{5}{|l|}{ Thickness (mm) } \\
\hline$\leq 1.00$ & $9(9.4)$ & $72(61.0)$ & $1^{\mathrm{b}}$ & $1^{\mathrm{b}}$ \\
\hline $1.01-2.00$ & $24(25.0)$ & $22(18.6)$ & $8.73(3.54-21.52)$ & $7.22(2.73-19.05)$ \\
\hline $2.01-4.00$ & $44(45.8)$ & $22(16.6)$ & $16.00(6.76-37.87)$ & $7.04(2.54-19.56)$ \\
\hline$>4.00$ & $19(19.8)$ & $2(1.7)$ & $76.00(15.14-381.51)$ & $51.78(5.65-474.86)$ \\
\hline$x^{2}$ trend: $p$ value & & & $p<0.0001$ & $p<0.0001$ \\
\hline \multicolumn{5}{|l|}{ Ulceration } \\
\hline Absent & $46(47.9)$ & $96(81.4)$ & $1^{\mathrm{b}}$ & $1^{\mathrm{b}}$ \\
\hline Present & $43(44.8)$ & $18(15.2)$ & $4.98(2.59-9.57)$ & $1.56(0.67-3.62)$ \\
\hline Unknown & $7(7.3)$ & $4(3.4)$ & & \\
\hline$x_{1}^{2}: p$ value & & & $p<0.0001$ & $p=0.3066$ \\
\hline \multicolumn{5}{|l|}{ Regression } \\
\hline Absent & $65(67.7)$ & $67(56.8)$ & $1^{\mathrm{b}}$ & $1^{\mathrm{b}}$ \\
\hline Present & $24(25.0)$ & $46(39.0)$ & $0.54(0.30-0.98)$ & $0.55(0.25-1.22)$ \\
\hline Unknown & $7(7.3)$ & $5(4.2)$ & & \\
\hline$x_{1}^{2}: p$ value & & & $p=0.0428$ & $p=0.1393$ \\
\hline \multicolumn{5}{|l|}{ Mitoses (n/mm²) } \\
\hline$\leq 2$ & $31(32.3)$ & $86(72.9)$ & $1^{\mathrm{b}}$ & $1^{\mathrm{b}}$ \\
\hline $3-5$ & $24(25.0)$ & $14(11.9)$ & $4.76(2.19-10.34)$ & $2.62(1.01-6.83)$ \\
\hline$>5$ & $35(36.5)$ & $11(9.3)$ & $8.83(4.00-19.49)$ & $4.87(1.77-13.40)$ \\
\hline Unknown & $6(6.2)$ & $7(5.9)$ & & \\
\hline$x^{2}$, trend: $p$ value & & & $p<0.0001$ & $p=0.0015$ \\
\hline \multicolumn{5}{|l|}{$\mathrm{TIL}^{\mathrm{d}}$} \\
\hline Absent & $29(30.2)$ & $34(28.8)$ & $1^{b}$ & \\
\hline Brisk & $14(14.6)$ & $27(22.9)$ & $0.61(0.27-1.37)$ & \\
\hline Non-Brisk & $46(47.9)$ & $48(40.7)$ & $1.12(0.59-2.13)$ & \\
\hline Unknown & $7(7.3)$ & $9(7.6)$ & & \\
\hline$x^{2}{ }_{1}$ trend: $p$ value & & & $p=0.6174$ & \\
\hline
\end{tabular}

Table 2 shows the univariate and multivariate analysis of recurrence (HR) of some histopathological features in patients with NM and SSM. Multivariate analysis showed that BT [BT 1.01-2.00 mm (HR 1.55; 95\% CI 0.51-4.71), BT 2.01-4.00 mm (HR 2.42; 95\% CI, 0.896.54), $\mathrm{BT}>4.00 \mathrm{~mm}$. (HR 3.13; 95\% CI 0.95-10.28) $(\mathrm{p}=0.05)], \mathrm{MR}>2$ mitoses $/ \mathrm{mm}^{2}$ (HR 2.34; 95\% CI $1.11-$ $4.97)(\mathrm{p}=0.03)$ and the positivity of sentinel lymph node biopsy (SNLB) (HR 2.60; 95\% CI 1.19-5.68) ( $\mathrm{p}=0.007)$ were significantly associated with an increased risk of recurrence (Table 2).

By contrast, the risk of progression was not significantly associated with NM histotype (Table 2).

The probability of recurrence according to $\mathrm{BT}$ and MR are reported in Fig. 1; at 5 years, the percentage of patients recurrence free were $45 \%$ (95\% CI 23-65), 56\% (95\% CI 43-67), 83\% (95\% CI 68-92), and 92\% (95\% CI 83-96) for patients with BT $>4.00 \mathrm{~mm}, 2.01-4.00 \mathrm{~mm}$, $1.01-2.00 \mathrm{~mm}$, and $\leq 1 \mathrm{~mm}$, respectively. In addition, the percentage of patients recurrence free were 55\% (95\% CI 44-65), and 89\% (95\% CI 81-94), for MR > 2, and $\leq 2$ mitoses $/ \mathrm{mm}^{2}$, respectively.

\section{Discussion}

The NM subtype accounts for a large amount of thick melanomas, representing from 40 to $65 \%$ of all $>2 \mathrm{~mm}$ thick melanomas $[6,11,12]$. In line with previous reports [6, 11-13], present results indicate that the NM subtype is significantly associated with higher BT and MR values at multivariate analysis. Furthermore, our results support 
Table 2 Univariate and multivariate analysis of recurrence (HR) of some histopathological features of patients with nodular melanoma (NM) and superficial spreading melanomas (SSMs)

\begin{tabular}{|c|c|c|c|c|}
\hline & \multicolumn{2}{|l|}{ Recurrence } & \multirow{2}{*}{$\begin{array}{l}\text { Univariate } \\
\text { HR }(95 \% \mathrm{Cl})^{\mathrm{a}}\end{array}$} & \multirow{2}{*}{$\begin{array}{l}\text { Multivariate }^{c} \\
\text { HR }(95 \% \mathrm{Cl})^{a}\end{array}$} \\
\hline & Yes (N. 53) & No (N. 161) & & \\
\hline \multicolumn{5}{|l|}{ Diagnosis } \\
\hline SSMs & $14(26.4)$ & $104(64.6)$ & $1^{\mathrm{b}}$ & $1^{\mathrm{b}}$ \\
\hline NM & $39(73.6)$ & $57(35.4)$ & $4.09(2.22-7.53)$ & $1.62(0.77-3.39)$ \\
\hline$x_{1}^{2}: p$ value & & & $p<0.0001$ & $p=0.2016$ \\
\hline \multicolumn{5}{|l|}{ Thickness (mm) } \\
\hline$\leq 1$ & $7(13.2)$ & $74(46.0)$ & $1^{b}$ & $1^{\mathrm{b}}$ \\
\hline $1.01-2.00$ & $7(13.2)$ & $39(24.2)$ & $2.07(0.72-5.89)$ & $1.55(0.51-4.71)$ \\
\hline $2.01-4.00$ & $28(52.8)$ & $38(23.6)$ & $5.85(2.55-13.41)$ & $2.42(0.89-6.54)$ \\
\hline$>4.00$ & $11(20.8)$ & $10(6.2)$ & $7.45(2.89-19.22)$ & $3.13(0.95-10.28)$ \\
\hline$x^{2}{ }_{1}$ trend: $p$ value & & & $p<0.0001$ & $p=0.0456$ \\
\hline \multicolumn{5}{|l|}{ Ulceration } \\
\hline Absent & $29(54.7)$ & $113(70.2)$ & $1^{\mathrm{b}}$ & $1^{\mathrm{b}}$ \\
\hline Present & $24(45.3)$ & $37(23.0)$ & $2.10(1.22-3.61)$ & $0.74(0.40-1.39)$ \\
\hline Unknown & - & $11(6.8)$ & & \\
\hline$x_{1}^{2}$ : p value & & & $p=0.0073$ & $p=0.3513$ \\
\hline \multicolumn{5}{|l|}{ Regression } \\
\hline Absent & $37(69.8)$ & $95(59.0)$ & $1^{2}$ & \\
\hline Present & $16(30.2)$ & $54(33.5)$ & $0.81(0.45-1.45)$ & \\
\hline Unknown & - & $12(7.5)$ & & \\
\hline$x^{2}$ : $p$ value & & & $p=0.4687$ & \\
\hline \multicolumn{5}{|l|}{ Mitoses $\left(\mathrm{n} / \mathrm{mm}^{2}\right)$} \\
\hline$\leq 2$ & $13(24.5)$ & $104(64.6)$ & $1^{\mathrm{b}}$ & $1^{\mathrm{b}}$ \\
\hline$>2$ & $37(69.8)$ & $47(29.2)$ & $4.75(2.52-8.94)$ & $2.34(1.11-4.97)$ \\
\hline Unknown & $3(5.7)$ & $10(6.2)$ & & \\
\hline$x_{1}^{2}: p$ value & & & $p<0.0001$ & $p=0.0263$ \\
\hline \multicolumn{5}{|l|}{$\mathrm{TIL}^{\mathrm{d}}$} \\
\hline Absent & $21(39.6)$ & $42(26.1)$ & $1^{\mathrm{b}}$ & \\
\hline Brisk & $9(17.0)$ & $32(19.9)$ & $0.62(0.29-1.36)$ & \\
\hline Non-Brisk & $22(41.5)$ & $72(44.7)$ & $0.64(0.35-1.16)$ & \\
\hline Unknown & $1(1.9)$ & $15(9.3)$ & & \\
\hline$x^{2}$, trend: $p$ value & & & $p=0.1527$ & \\
\hline \multicolumn{5}{|c|}{ Lymph nodes sentinel } \\
\hline No suggest & $15(28.3)$ & $81(50.3)$ & $1^{\mathrm{b}}$ & $1^{\mathrm{b}}$ \\
\hline Yes-negative & $16(30.2)$ & $66(41.0)$ & $1.14(0.56-2.31)$ & $0.77(0.35-1.69)$ \\
\hline Yes-positive & $22(41.5)$ & $14(8.7)$ & $5.24(2.71-10.13)$ & $2.60(1.19-5.68)$ \\
\hline$x^{2}$ trend: $p$ value & & & $p<0.0001$ & $p=0.0073$ \\
\hline
\end{tabular}

${ }^{a}$ Hazard ratio and $95 \%$ confidence interval (Cl)

b Reference category

c Model including all significant terms in the univariate analysis

d Tumor infiltrating lymphocytes

previous observations that NM presents with higher MR values than SSM; indeed, the median number of mitoses reported for NM vs SSM was 2.5 and $1 / \mathrm{mm}^{2}$ respectively [13]. Different features of the two melanoma subtypes may derive from distinct genetic pathways and different origin $[7,14]$. One could speculate that the hypothesized origin of NM from dermal stem cells and SSM from epidermal stem cells [14] could explain behavioral differences between the two melanoma subtypes. In particular, the growth of NM much faster than that of SSM should be considered as a feature closely associated with higher BT and MR scores among NM lesions [3]. 

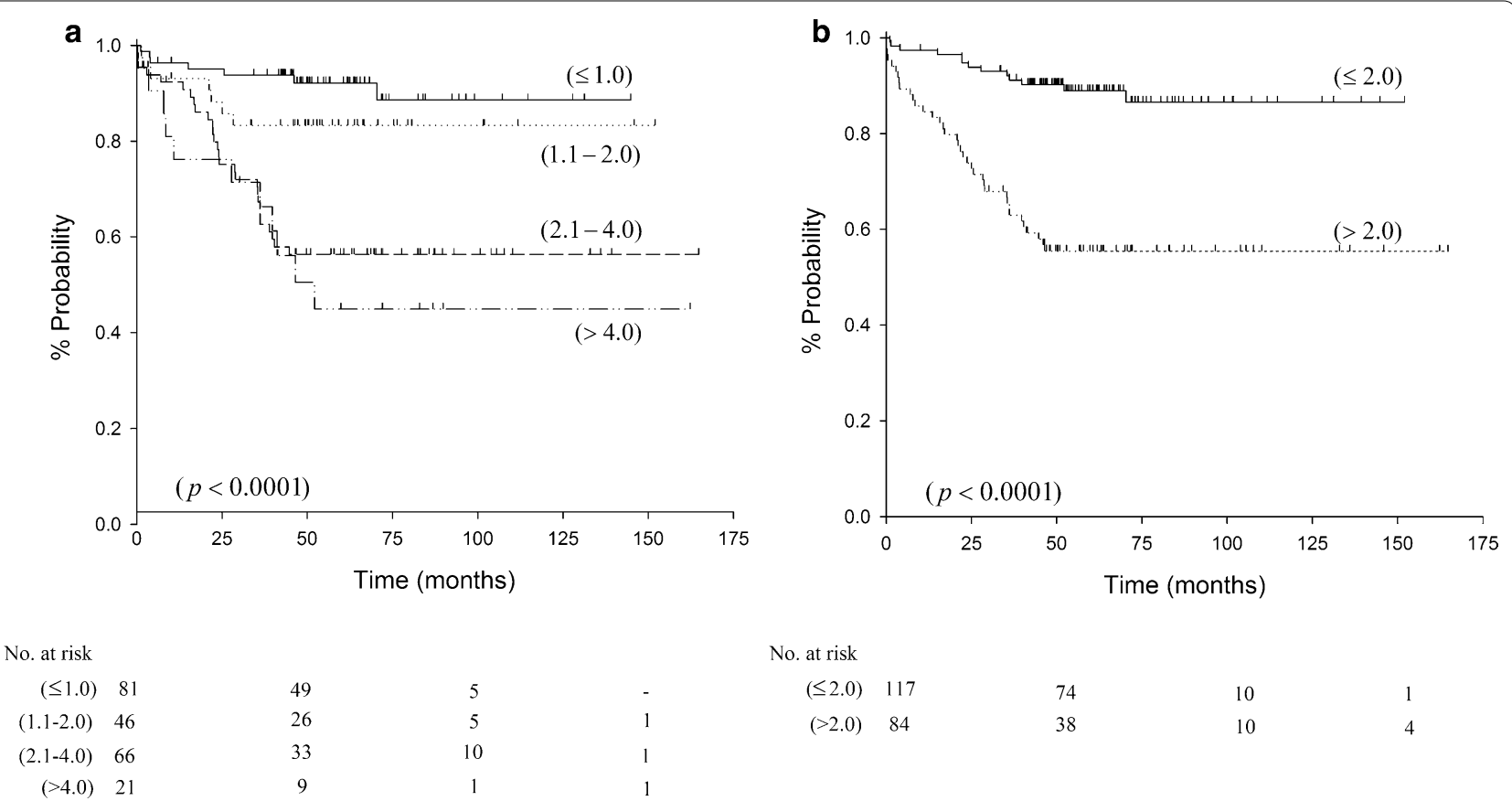

$\begin{array}{cc}49 & 5 \\ 26 & 5 \\ 33 & 10 \\ 9 & \end{array}$

5
5
10
1

No. at risk

$(\leq 2.0) \quad 117$

$(>2.0) \quad 84$

Fig. 1 Probability of recurrence according to thickness $(\mathrm{mm})(\mathbf{a})$ and mitotic rate $\left(\mathrm{n} . / \mathrm{mm}^{2}\right)(\mathbf{b})$ in 214 cutaneous melanoma patients

By multivariate analysis, ulceration and regression were not significantly more frequent in NM as compared with SSM. In agreement with our results, Warycha et al. [13] did not find any significant difference between NM and SSM regarding the presence of regression. In contrast with our findings, this study however reported a significantly higher probability for NM to be found ulcerated [13].

No statistically significant difference was found in NM vs SSM in relation to tumor infiltrating lymphocytes (TILs) in the univariate analysis. This result is in contrast with that of other studies showing that patients with TILs more likely had nodular histology [15].

A limitation to our retrospective study was the selection bias and institutional variation in pathology assessment that could have potentially influenced our results.

NM was more frequently diagnosed in older men ( $\geq 50$ years), particularly on the lower limbs or head and neck regions [6]. Consistently with Warycha et al. [13] study, we did not find any differences between NM and SSM regarding sex, age, and anatomical site (data not shown).

In agreement with prior reports [16-18], increasing BT, MR with $>2$ mitoses $/ \mathrm{mm}^{2}$, and the positivity of SNLB were significantly associated with an increased risk of recurrence in all melanoma cases (regardless of the subtype, NM or SSM). However, MR can be associated with SNLB positivity as reported by Mandalà et al.
[19], whose study found that MR $>1$ mitoses $/ \mathrm{mm}^{2}$ in primary cutaneous melanoma with $\mathrm{BT} \leq 1 \mathrm{~mm}$ was significantly predictive of metastasis in corresponding sentinel lymph node. The greater probability of recurrence with increasing BT from T1 to T4 and MR > 2 mitoses $/ \mathrm{mm}^{2}$ is reported in Fig. 1.

In our study, NM was associated with a significant higher risk of recurrence in the univariate analysis but the association became not significant in the multivariate analysis. By contrast, Faut et al. [20] found that SLNB-negative patients with NM had a significant higher recurrence rate in the multivariate analysis (HR 1.82, $\mathrm{p}=0.028$ ). However, in our study, the HR was similar (1.62) but with a higher $\mathrm{CI}$, resulting in a not significant $\mathrm{p}$ value. In addition, in Faut et al. [20] study, the significant association between $\mathrm{NM}$ and higher risk of recurrence was found solely in SLNB-negative and not in SLNBpositive patients. The differences between the two studies could depend on the different sample size of both NMs and SSMs.

O' Connel et al. [21] have also reported a significant association between NM subtype and melanoma recurrence in patients with negative SLNB. However, in this latter study only SLNB- negative patients were included, while our series comprised both SLNB negative and positive patients [21].

In addition, and consistently with our results, other Authors found that NM was not significantly associated 
with higher risk recurrence in the multivariate analysis [22]. Although SSM has been found to have a better prognosis than NM, after adjusting for BT, a significant difference between two subtypes was not found [8]. Poor prognosis of NM could depend on a greater $\mathrm{BT}$, as a consequence of a delayed diagnosis and not due to an intrinsic malignant effect of NM.

In our study, a significant association between ulceration and increased risk of recurrence was observed only in the univariate but not in the multivariate analysis, in agreement with previous studies that did not show an independent significant effect of ulceration on prognosis [17].

In addition, we did not find a statistically significant correlation between regression and recurrence in the univariate analysis. According to Tas et al. [23], the presence of histological regression plays no significant prognostic role in melanoma patients. However, the meta-analysis of 10 studies proposed that the regression can be considered a protective factor, probably depending on early activation of the host immune system against melanoma [24]. The prognostic value of histologic regression in melanoma remains controversial possibly due to lack of a standardized definition and objective criteria for histopathological classification of regression-associated parameters.

In our study, TIL response was not significantly associated with recurrence in the univariate analysis. In previous studies, TIL response was reported as an important independent prognostic indicator and the absence of TIL was considered as an independent predictor of SNL metastasis in melanoma [25].

We cannot exclude that the limited sample size, the participation of numerous centers in absence of lack of central histopathology review, as well as the relative short follow-up may account for this negative result. In multicentric studies, improved standardization in density and distribution of TILs is essential before the biologic and prognostic significance of histologic parameters related to immunity such as regression and TILs can be recognized.

Unfortunately, the limited series of cases in our study did not allow to rule out that NM could represent an independent prognostic factor.

\section{Conclusions}

In summary, we herein showed that NM subtype was significantly associated with higher BT and MR scores. The NM subtype per se was not a prognostic factor, since it did not significantly affect the melanoma recurrence rate; the risk of melanoma recurrence was instead increased by higher BT, MR $>2 / \mathrm{mm}^{2}$, and SNLB positivity. Our study limitations did not allow drawing firm conclusions on the prognostic role of the NM subtype.

\section{Abbreviations}

NM: nodular melanoma; SSM: superficial spreading melanoma; BT: breslow thickness; MR: mitotic rate; SNLB: lymph node sentinel biopsy; LMM: lentigo maligna melanoma; TIL: tumour infiltrating lymphocytes.

\section{Authors' contributions}

The authors' responsibilities were as follows: MAP, DM, GP were responsible for the concept and design of the study; MAP, DM and MM helped interpret the finding and contributed to the writing of the manuscript; MAP, PQ, VDG, GG, SC, PQ, MTC, PR, MA, SA, FA, SM, LM, MAM contributed to the acquisition and interpretation of data; DS and RT conducted statistical analyses; MAP, DM, MM, $\mathrm{PQ}$ and GP provided a critical review of the manuscript. All authors read and approved the final manuscript.

\section{Author details}

${ }^{1}$ Division of Oncology B, CRO Aviano National Cancer Institute, Via Franco Gallini 2, 33081 Aviano, Italy. ${ }^{2}$ Division of Pathological Anatomy, Department of Surgery and Translational Medicine, University of Florence, Florence, Italy. ${ }^{3}$ Unit of Medical Oncology, Papa Giovanni XXIII Hospital, Bergamo, Italy. ${ }^{4}$ Department of Medical Oncology, National Institute for Cancer Research, IRCCS San Martino, Genoa, Italy. ${ }^{5}$ Skin Cancer Unit, Istituto Tumori Romagna (IRST), Meldola, Italy. ${ }^{6}$ Department of Dermatology, University of Florence, Florence, Italy. ${ }^{7}$ Clinic of Dermatology, IRCCS San Martino-IST, Genoa, Italy. ${ }^{8}$ Department of Dermatology, Fondazione Ospedale Maggiore Policlinico IRCCS, Milan, Italy. ${ }^{9}$ Dermatologic Clinic, Dept Medical Sciences, University of Torino, Turin, Italy. ${ }^{10}$ Division of Dermatology, Pordenone Hospital, Pordenone, Italy. ${ }^{11}$ Department of Dermatology, University of Siena, Siena, Italy.

${ }^{12}$ Department of Dermatology, University of Padova, Padua, Italy. ${ }^{13}$ Division of Dermatology, Celio Hospital, Rome, Italy. ${ }^{14}$ National Cancer Institute, "Fondazione G. Pascale"-IRCCS, Naples, Italy. ${ }^{15}$ Department of Dermatology, ASST degli Spedali Civili di Brescia, Brescia, Italy. ${ }^{16}$ Unit of Epidemiology and Biostatistics, CRO Aviano National Cancer Institute, Aviano, Italy. ${ }^{17}$ Unit of Cancer Genetics, Institute of Biomolecular Chemistry (ICB), National Research Council (CNR), Sassari, Italy. ${ }^{18}$ Department of Dermatology, University of Parma, Parma, Italy.

\section{Acknowledgements}

The authors wish to thank Luigina Mei for her editing assistance.

\section{Competing interests}

The authors declare that they have no competing interests.

\section{Availability of data and materials}

The datasets analysed during the present study are available from the authors (MAP, DS and RT) on reasonable request.

\section{Consent for publication}

Not applicable.

\section{Ethical approval and consent to participate}

Given the descriptive nature of the study, the approval of Ethical Committee was not required.

Funding sources

None.

\section{Publisher's Note}

Springer Nature remains neutral with regard to jurisdictional claims in published maps and institutional affiliations.

Received: 24 July 2017 Accepted: 27 October 2017

Published online: 07 November 2017

\section{References}

1. Fuglede NB, Brinck-Claussen UO, Deltour I, Boesen EH, Dalton SO, Johansen C. Incidence of cutaneous malignant melanoma in Denmark 1978-2007. Br J Dermatol. 2011;2:349-53. 
2. UICC-TNM classification of malignant tumours. Eight edition, edited by Brierlev JD, Gospodarowicz MK and Wittekind C, 2017.

3. Liu W, Dowling JP, Murray WK, McArthur GA, Thompson JF, Wolfe R, et al. Rate of growth in melanomas: characteristics and associations of rapidly growing melanomas. Arch Dermatol. 2006;142:1551-8.

4. Bastian BC. The molecular pathology of melanoma: an integrated taxonomy of melanocytic neoplasia. Annu Rev Pathol. 2014;9:239-71.

5. Grob JJ, Richard MA, Gouvernet J, Avril MF, Delaunay M, Wolkenstein P, et al. The kinetics of the visible growth of a primary melanoma reflects the tumor aggressiveness and is an independent prognostic marker: a prospective study. Int J Cancer. 2002;102:34-8.

6. Demierre MF, Chung C, Miller DR, Geller AC. Early detection of thick melanomas, in the United States: beware of the nodular subtype. Arch Dermatol. 2005;141:745-50.

7. Shaikh WR, Xiong M, Weinstock MA. The contribution of nodular subtype to melanoma mortality in the United States, 1978-2007. Arch Dermatol. 2012;148:30-6.

8. Wisco OJ, Sober AJ. Prognostic factors for melanoma. Dermatol Clin. 2012;30:469-85

9. Kaplan EL, Meier P. Non-parametric estimation from incomplete observations. J Am Stat Assoc. 1958;53:457-81.

10. Cox DR. Regression model and life-tables. J R Stat Soc B. 1972;34:178-220.

11. Bergenmar M, Hansson J, Brandberg Y. Detection of nodular and superficial spreading melanoma with tumor thickness $\leq 2.00 \mathrm{~mm}$-an interview study. Eur J Cancer Prev. 2002;11:49-55.

12. Carli P, De Giorgi V, Palli D, Maurichi A, Mulas P, Orlandi C, et al. Patterns of detection of superficial spreading and nodular-type melanoma: multicenter Italian study. Dermatol Surg. 2004;30:1371-6.

13. Warycha MA, Christos PJ, Mazumdar M, Darvishian F, Shapiro RL, Berman RS, et al. Changes in the presentation of nodular and superficial spreading melanomas over 35 years. Cancer. 2008;113:3341-8.

14. Zalaudek I, Marghoob AA, Scope A, Leinweber B, Ferrara G, Hofmann-Wellenhof $\mathrm{R}$, et al. Three roots of melanoma. Arch Dermatol. 2008;144:1375-8.

15. Tas F, Erturk K. Tumour infiltrating lymphocytes (TILs) may be only an independent predictor of nodal involvement but not for recurrence and survival in cutaneous melanoma patients. Cancer Invest. 2017;35:501-5.

16. Egger ME, Dunki-Jacobs EM, Callender GG, Quillo AR, Scoggins CR, Martin RCG II, et al. Outcomes and prognostic factors in nodular melanomas. Surgery. 2012;152:652-60
17. Deshmane V, Kalloli M, Chikaraddi S, Keerthi BR, Krishnappa R, et al. Predictive factors for loco regional recurrence and distant metastasis following primary surgical treatment of cutaneous melanoma. Indian J Dermatol (Dermatol Intern Med). 2014;59:241-6.

18. Matheson JAH, te Marvelde L, Mailer S, Speakman D, Spillane J, Henderson MA, et al. Prospective evaluation of prognostic indicators for early recurrence of cutaneous melanoma. Melanoma Res. 2016. https://doi. org/10.1097/CMR.0000000000000302.

19. Mandala'M, Galli F, Cattaneo L, Merelli B, Rulli E, Ribero S, et al. Mitotic rate correlates with sentinel lymph node status, and out come in cutaneous melanoma greater than $1 \mathrm{~mm}$ in thickness: a multi-institutional study of 1524 cases. J Am Acad Dermatol. 2017;76:264-73.

20. Faut M, Wevers KP, Van Ginkel RJ, Diercks GFH, Hoekstra HJ, Kruijff S, et al. Nodular histologic subtype and ulceration are tumor factors associated with high risk of recurrence in sentinel node-negative melanoma patients. Ann Surg Oncol. 2016. https://doi.org/10.1245/ s10434-016-5566-8.

21. O'Connel EP, O'Leary DP, Fogarty K, Khan ZJ, Redmond HP. Predictors and patterns of melanoma recurrence following a negative sentinel lymph node biopsy. Melanoma Res. 2016:26:66-70.

22. Brauer JA, Wriston CC, Troxel AB, Elenitsas R, Shin DB, Guerry D, Ming ME. Characteristics associated with early and late melanoma metastases. Cancer. 2010;116:415-23.

23. Tas F, Erturk K. Presence of histological regression as a prognostic factor in cutaneous melanoma patients. Melanoma Res. 2016. https://doi. org/10.1097/CMR.0000000000000277.

24. Gualano MR, Osella A, Scaioli G, Marra E, Bert F, Faure E, Baduael ES, Balagna E, Quaglino P, Fierro MT, Siliquini R, Ribero S. Prognostic role of histologic regression in primary cutaneous melanoma: a systematic review and Meta-analysis. Br J Dermatol. 2017. https://doi.org/10.1111/ bjd.15552.

25. Azimi F, Scolyer RA, Rumcheva P, Moncrieff M, Murali R, Mc Carthy SW, Saw RP, Thompson JF. Tumour-infiltrating lymphocyte grade is an independent predictor of sentinel lymph node status and survival in patients with cutaneous melanoma. J Clin Oncol. 2012:30:2678-83. https://doi. org/10.1200/JCO.2011.37.8539.

\section{Submit your next manuscript to BioMed Central and we will help you at every step:}

- We accept pre-submission inquiries

- Our selector tool helps you to find the most relevant journal

- We provide round the clock customer support

- Convenient online submission

- Thorough peer review

- Inclusion in PubMed and all major indexing services

- Maximum visibility for your research

Submit your manuscript at www.biomedcentral.com/submit
BioMed Central 